\title{
Observing CVs and LMXBs with SALT: Updates and Recent Results
}

\author{
David A. H. Buckley ${ }^{1}$ \\ ${ }^{1}$ Southern African Large Telescope, c/o South African Astronomical Observatory, PO Box 9, Observatory 7935, Cape \\ Town, South Africa
}

Corresponding author: dibnob@salt.ac.za

\begin{abstract}
I present an overview of the ongoing observational programs utilizing the Southern African Large Telescope (SALT), focussing on magnetic Cataclysmic Variables and Low Mass X-ray Binaries. SALT's instruments and capabilities are well suited to time resolved studies of the accretion phenomena exhibited in these systems. Initial observations, using SALTICAM, have been used to derive high time resolution $(\sim 100 \mathrm{~ms})$ eclipse light curves, with high signal-to-noise, of Polars. Recently this work has been extended to time resolved spectroscopic studies, utilizing the SALT Robert Stobie Spectrograph (RSS), allowing an opportunity to probe how the emission lines change during eclipse. A program to search for and characterize quasi-periodic oscillations (QPOs) in magnetic CVs using the SAAO 1.9-m telescope, begun in 2012, has been expanded to include observations with a photon counting camera (BVIT) on SALT. A multi-longitude campaign involving SALT, other SAAO facilities plus the ESO NTT, was carried out in March/April 2012 on the enigmatic gamma ray source, XSS J12270-4859, which has revealed the that system exhibits spectral line variations, from absorption to emission, seemingly over timescales of $<1 \mathrm{~h}$.
\end{abstract}

Keywords: cataclysmic variables - polars - intermediate polars - optical - spectroscopy - photometry - X-rays - individual: FL Cet, EX Hya, HU Aqr, XSS J12270-4859.

\section{Introduction}

The Southern African Large Telescope (SALT) is one of five 10-m class segmented mirror telescopes and the only one situated in the southern hemisphere. At the last Palermo CV meeting in 2011, I summarized the capabilities and the status at that time (Buckley 2012) and presented some early science results. Since then SALT has entered full time science operations (in November 2011) and has begun to steadily increase its scientific productivity (up to $\sim 60$ refereed publications).

There are still some remaining issues to resolve, however, notably implementation of a mirror edge sensing system to allow active control of the primary mirror array. This is currently under development and the first batch of new inductive edge sensors will be installed early in 2014, while the final ones will be installed late in 2015. This will lead to much improved image quality and, more importantly its retention during an observation. Similarly there are improvements being made to the throughput of the main work-horse SALT science instrument, the Robert Stobie Spectrograph (RSS), which should greatly improve its performance, particularly in the blue $(320-400 \mathrm{~nm})$. This is being achieved by fabrication of new collimator optics and opto-mechanics, designed to avoid the optical fluid contamination problems that have led to the current throughput under-performance. These new optics are expected to be installed by mid- 2014 .

SALT is a $100 \%$ queue-scheduled telescope, where all observations are undertaken in a service observing mode by SALT staff. This modus operandi lends itself to target-of-opportunity observations and synoptic monitoring campaigns, both of which are often appropriate for observations of Cataclysmic Variables and related objects. For example, to cover specific phase intervals, to coordinate with X-ray or other satellite observations, to monitor a system through an outburst or accretion state change, etc. Just such observations have already been carried out with SALT and some examples are discussed in this paper.

\subsection{High speed photometry with SALTICAM}

SALTICAM employs frame transfer (FT) CCDs, which allows for fast acquisition and imaging. It also has a moving occulting mask which allows for 3 imaging modes: full field imaging, frame transfer mode (half of the field is imaged) and a specialized high-speed mode, 
where a narrow slot is imaged. The latter, known as "slot mode", allows for high-speed (up to $\sim 12.5 \mathrm{~Hz}$ ) photometry and has been used extensively for observing $\mathrm{CVs}$, X-ray binaries and related systems.

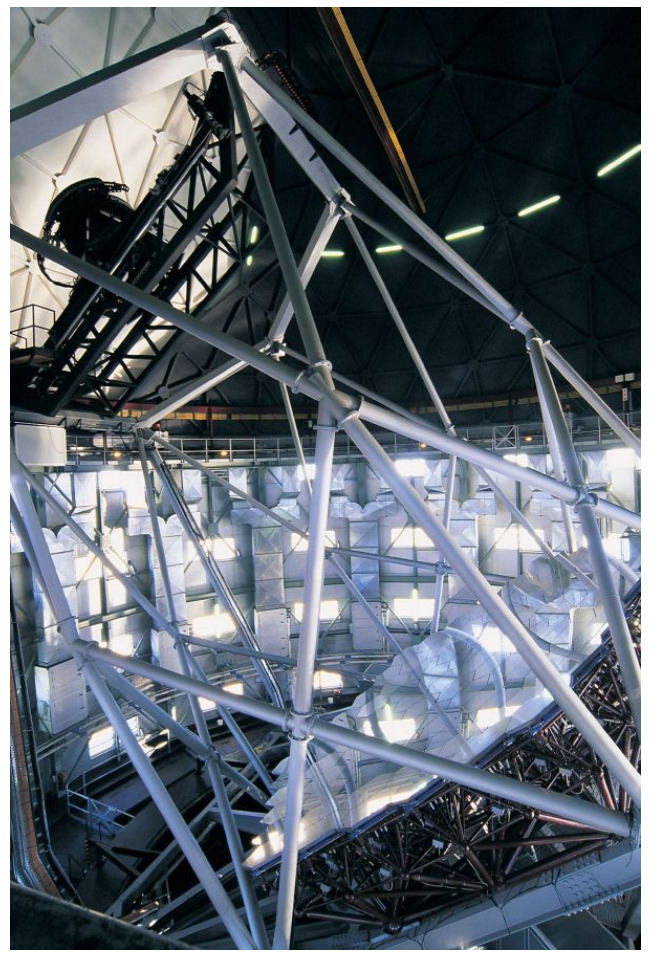

Figure 1: The 11-m Southern African Large Telescope (SALT), consisting of $91 \times 1.2 \mathrm{~m}$ (corner-to-corner) hexagonal mirror segments.

This mode allows for just 144 rows of the CCD mosaic to be illuminated, equivalent to $\sim 20$ arcsec $\times 10$ arcmin rectangular region on the sky. The object of interest, plus ideally a nearby $(<$ a few arcmin) constant source (i.e. a comparison star), are placed in the centre of this slot, which can be achieved by rotating the instrument to the desired position angle. The illuminated region of the CCD is then exposed (typically with exposure times ranging from $80-1000 \mathrm{~ms}$ ) and then quickly (within a few ms) row-shifted into the storage array, while another exposure begins. Thus exposed slot regions are sequentially moved through the storage array to the serial readout register. During the next exposure, the latest exposed region to reach the bottom of the chip is readout in a time $<$ the exposure time, which is dependent on the on-chip binning factor (usually $4 \times 4$ pixels).

\subsection{Time resolved spectroscopy with RSS}

Like for all current SALT instruments, the Robert Stobie Spectrograph (RSS) resides at SALT's prime focus (f/4.2). The advantages of this include maximizing the performance at short wavelengths $(<400 \mathrm{~nm})$, by avoiding use of optical fibres, and ability to conduct FabryPerot imaging spectroscopy. RSS was designed to have a range of capabilities and observing modes, each one remotely and rapidly reconfigurable.

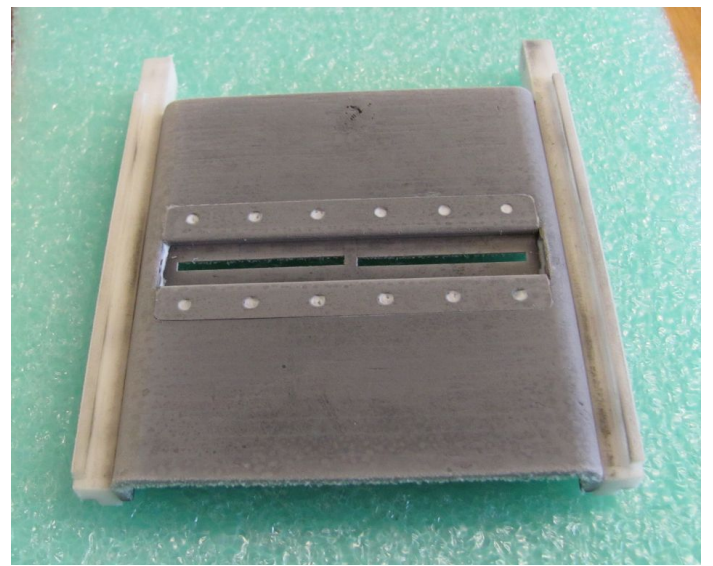

Figure 2: The narrow occulting slot that can be moved just in front of the SALTICAM CCDs to allow for high time resolution (down to $\sim 80 \mathrm{~ms}$ ) photometry.

In keeping with the overall philosophy of exploiting those areas where SALT has a competitive edge, the instrument has a range of capabilities, many well suited to $\mathrm{CV}$ and LMXB studies, including:

- Wavelength coverage from 320 to $900 \mathrm{~nm}$ for the initial UV-VIS configuration (a second near IR arm, extending to $1.7 \mu \mathrm{m}$, is under construction). Low to medium resolution spectroscopy (up to $\mathrm{R} \sim 6000$ with 1 arcsec slits) using efficient and tuneable VPH gratings.

- Narrow band, tuneable filter and Fabry-Perot imaging.

- High time resolution $(\sim 0.1 \mathrm{sec})$ spectroscopic and polarimetric modes.

- Imaging and spectropolarimetry (linear, circular and all Stokes modes), time resolved modes plus low resolution $(\mathrm{R} \sim 50)$ wide-field imaging spectropolarimetry.

High time resolution spectroscopy is achieved using a similar slot-mode option, as for SALTICAM. However, rather than the mask being just above the detector, it is placed at the telescope's focal plane, coplanar with the spectrograph's slit. This allows for the spectrum of the variable target to be imaged in a narrow rectangular region and then shuffled into the storage array at the end of the exposure. Three $2 \mathrm{k} \times 4 \mathrm{k}$ CCDs are mosaiced to allow spectra to be obtained over the entire 8 arcmin FoV (e.g. for MOS). Figure 3 shows the arrangement of 
the slot with respect to the CCD mosaic, where the slot is placed just above the imaging / storage boundary.

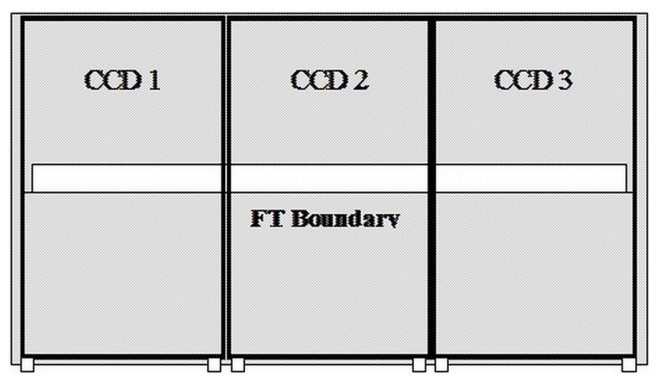

Figure 3: Schematic showing the region on the CCD where time resolved RSS spectra are recorded using a focal plane mask together with the spectrograph slit. Spectra of the target and sky are obtained in the rectangular region, which shuffled down across the frame transfer boundary at the end of a short exposure.

\section{Time-Resolved Studies of Polars}

The first high-speed observations conducted with SALT were mostly of eclipsing magnetic cataclysmic variable stars (mCVs), specifically polars. These systems emit the bulk of their luminosity (from X-rays to the optical / IR) from small accretion regions near the magnetic pole(s) of a strongly magnetized $\left(\sim 10^{1}\right.$ to $10^{2}$ MG) white dwarf. The dominant emission components in such systems includes hard $(>10 \mathrm{keV})$ thermal bremsstrahlung X-rays from an accretion shock just above the white dwarf surface. A fraction of this radiation is reprocessed in the white dwarfs photosphere into softer X-rays, UV and optical radiation. In some systems, ballistic "blobs" of accreting material bury into the white dwarf's photosphere, thermalize and contribute to the soft X-ray/extreme UV component. Shock cooling occurs through polarized optical/IR cyclotron emission. Polars therefore present a wealth of multi-wavelength observational opportunities, which are being exploiting with SALT.

\subsection{Light curves of eclipsing polars}

In eclipsing polars, the size, structure and location of accretion regions can be determined from high time resolution (sub-second) photometry. Since 2005 observations have been carried out with SALTICAM to obtain continuum eclipse curves with unprecedented time resolution and signal to noise.

An example is for the eclipsing system FL Cet, with a $1.45 \mathrm{~h}$ orbital period (O'Donoghue et al. 2006). An eclipse egress light curve, taken with SALTICAM in "slotmode" with $112 \mathrm{~ms}$ data sampling, is shown in Fig. 4. The intensity steps are due to the progres- sive re-appearance of two accretion hot-spots near the magnetic poles, which take $\sim 1.5 \mathrm{~s}$ to be uncovered. These data have been fitted with a model based on the likely masses of the component stars, as derived from the eclipse parameters and orbital period, plus the orbital inclination of the system $(i)$ and the co-latitudes of the accretion spots $\left(\beta_{1}\right.$ and $\left.\beta_{2}\right)$.

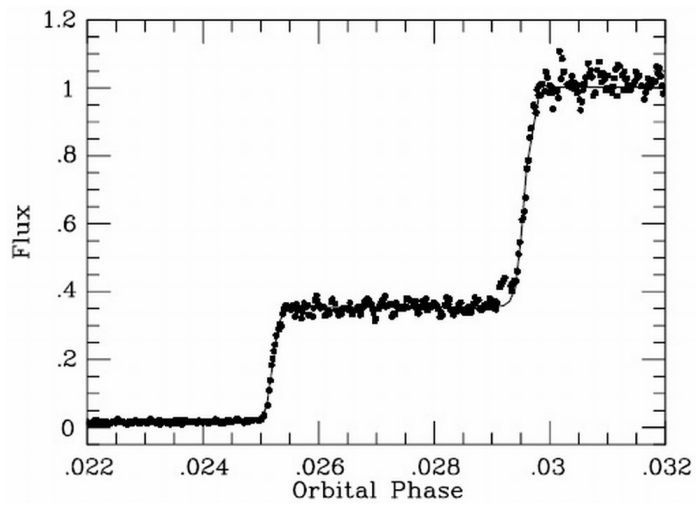

Figure 4: SALTICAM eclipse egress light curve of FL Cet, showing the re-appearance of two accretion spots.

Other Polars are also being systematically observed in order to derive similar quality light curves from which to determine the accretion geometries. An example is shown below for the system HY Eri, which has been observed in both high and low accretion states.

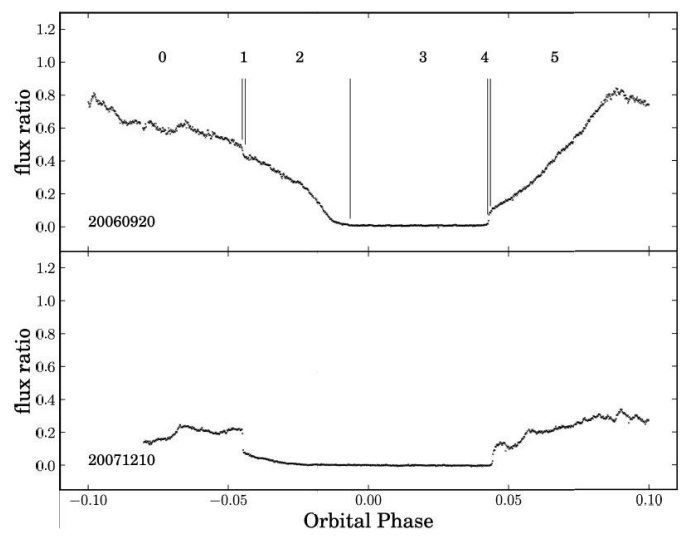

Figure 5: Eclipse light curves of HY Eri, at high (upper curve) and low (lower curve) accretion states.

\section{$2.2 \quad$ Spectra of eclipsing polars}

Since 2011 the eclipse light curve program has been extended to spectroscopic investigations of the emission lines of eclipsing polars, using RSS in time-resolved spectroscopy mode. The aim of these observations is to probe the mass transfer flow from the secondary star to the white dwarf, specifically where it changes from a 
ballistic to a magnetically controlled trajectory. The geometry of this so-called "threading region" varies from system to system and is dependent on the accretion rate and magnetic field topology. Previous attempts at probing the nature of the threading region (e.g. Hakala et al. 2002, Bridge et al. 2004) have often been frustrated by the problem of unambiguously determining the accretion stream structures from the 1-D data derived from broad band eclipse light curves.

These new SALT observations are aimed to address this issue and resolve details of the mass transfer stream through high time resolution emission line spectroscopy. Such data will lead to stream eclipse light curves as a function of wavelength and hence radial velocity, enabling the structure of the mass transfer stream to be determined. Doppler tomographic techniques will be applied to these data in order to attempt to map the positions of the line emitting regions. Recently a new "inside-out" Doppler tomography technique has been devised (e.g. see Kotze \& Potter; these proceedings). Eventually we plan to undertake phase resolved spectropolarimetric observations of these systems, using RSS (when the polarimetric modes are re-activated in 2014). These observations will probe the phase dependent cyclotron emission, giving us an independent view of the polarized emission arising from the accretion spots. During low states it may also be possible to study the polarization of the Zeeman lines from the white dwarf and the accretion halo.

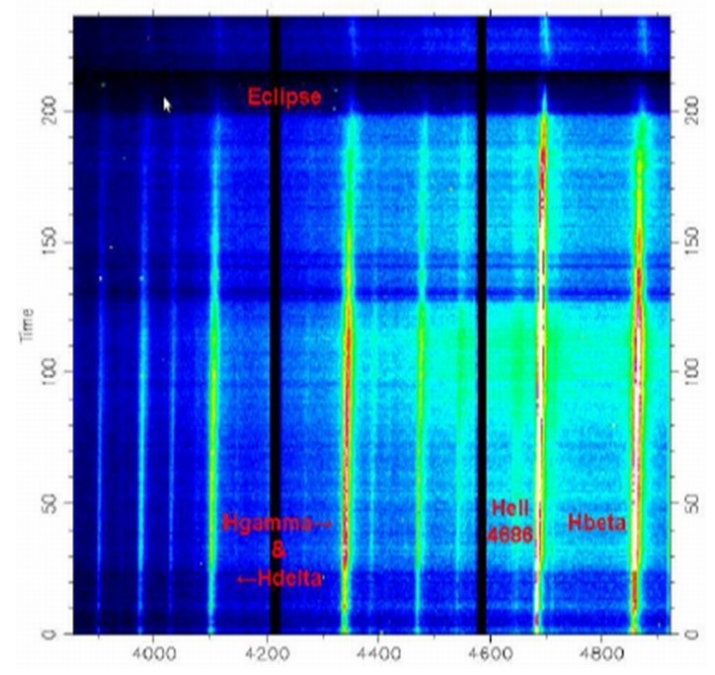

Figure 6: An example of time resolved (10s) trailed spectroscopy of the eclipsing polar HU Aqr.

\subsection{QPOs in magnetic CVs}

In 2012 a program to study quasi-periodic oscillations (QPOs) in magnetic CVs was instigated at SAAO, mostly using the new Electron Multiplication CCD
(EMCCD) cameras (SHOC) on the SAAO 1.9-m telescope. The aim is to search for and characterise the nature of the optical QPOs, which have typical periods of a few seconds. The commonly held understanding is that they are due to plasma oscillations in the magnetically confined accretion columns which constrain the accreted plasma before it cools and settles onto the surface of the magnetic white dwarf. The QPO frequency is thought to be related to the physical parameters inside the column (or sub-columns), particularly the shock height, accretion rate and magnetic field strength. This work will hopefully lead to a better understanding of the physics underlying the QPOs, taking the study from being purely phenomenological to one based on quantitative physical parameters. In conjunction with this observational program, we expect that the experimental results from a parallel program of simulating accreting columns in the laboratory will yield further relevant insights (e.g. Michaut et al. 2012).

The project involves new technology detectors, namely EMCCDs, which have both good QE and fast timing capability (e.g. $\sim 10 \mathrm{~ms}$ ). These detectors are superior to those used in the first studies of polar QPOs (e.g. Larsson 1989, and references therein), which relied on PMT (photomultiplier tube)-based instruments.

High time resolution photometry of mCVs have recently been carried out at SALT, utilizing the newly installed (in 2012) Berkeley Visible Image Tube (BVIT), a photon counting instrument using a Microchannel Plate (MCP) detectors (Buckley et al., 2010, Welsh et al. 2011). While not of particularly high QE, compared to a CCD, BVIT is capable of time tagging photons to $\sim 50 \mathrm{~ns}$, and is ideal for studying extremely fast variability (e.g. pulsars). Short (typically $\sim 1$ h) BVIT observations have been made of several $\mathrm{mCVs}$, but the only positive detection to date has been for the Intermediate Polar, EX Hya, where a 100 s QPO (and possibly its harmonic), were detected (Fig. 7).

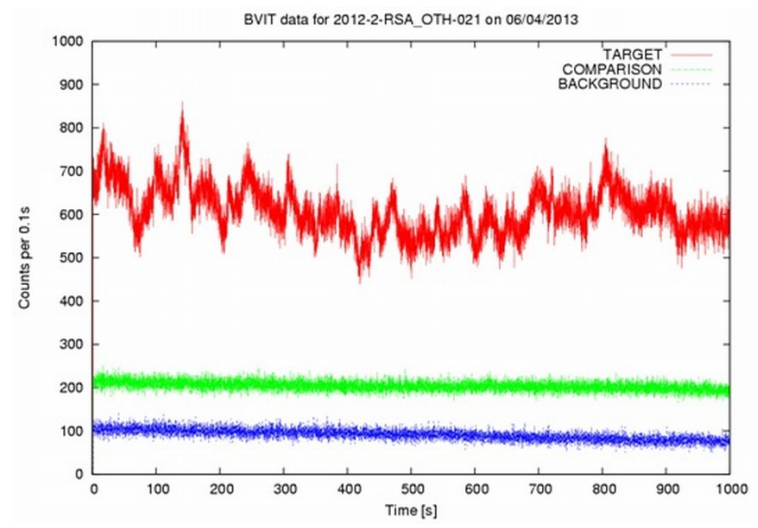

Figure 7: A SALT observations of EX Hya using BVIT which revealed a $\sim 100 / 50$ s QPO. 


\section{SALT Spectroscopy of XSS J12270-4859}
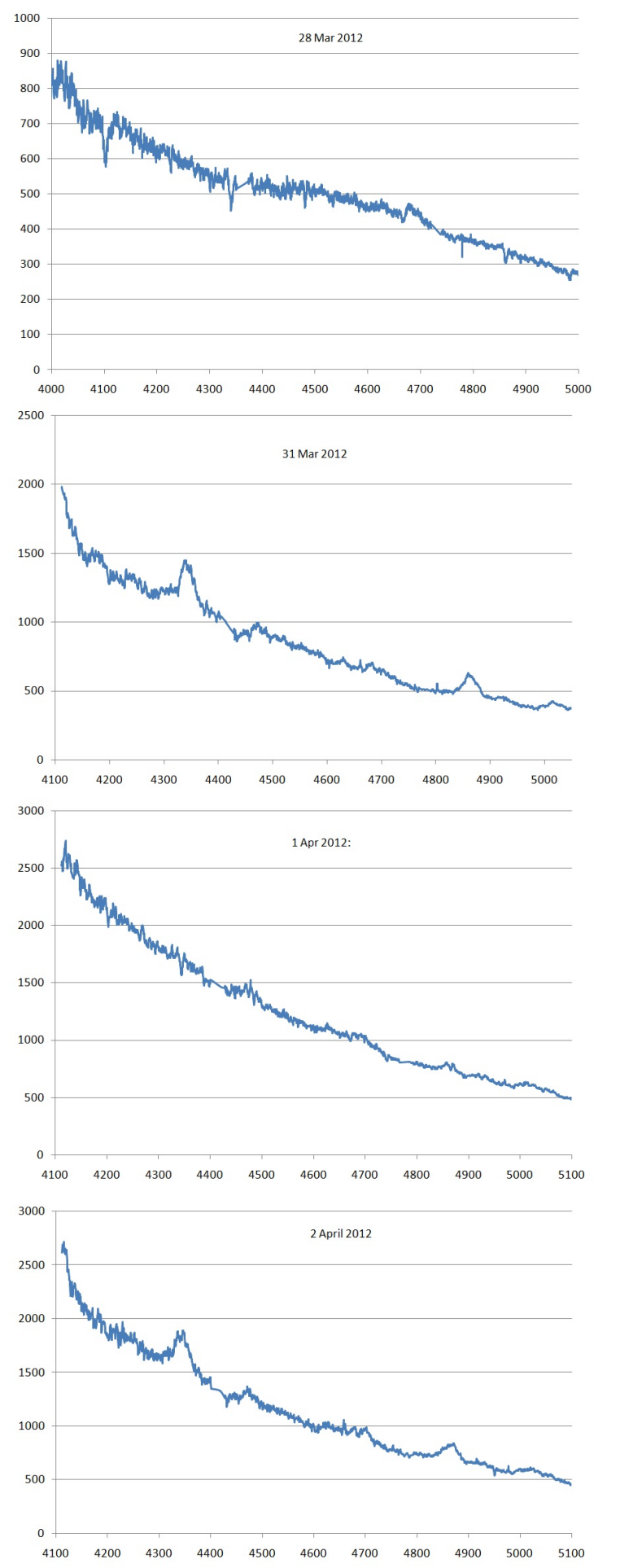

Figure 8: SALT spectra of XSS J12270-4859, averaged over $\sim 1$ h on 4 different nights.

The enigmatic FERMI gamma ray source, XSS J122704859 (e.g. Bonnet-Bidaud et al. 2012, and refer- ences therein), was the subject of a SAAO-ESO observing campaign in March/April 2012, utilizing the NTT, SALT and several smaller SAAO telescopes. Spectroscopy was carried out with SALT over 6 nights, contemporaneous with NTT spectroscopy. In addition, high speed $U$-band photometry and JHK imaging was also undertaken.

XSS J12270-4859 was first suggested to be an Intermediate Polar (Butters et al. 2008), but this was not confirmed from follow-up Suzaku and XMM observations. The system exhibits X-ray flares and dips, somewhat reminiscent of EXO0748-676, but without any phase dependency and no spectral changes. The $\mathrm{X}$-ray spectrum is also characterized by a weakly absorbed power law, with no cut-off up to $100 \mathrm{keV}$ and no Fe lines. The system has been suggested to be similar to a microquasar (e.g. Cyg X-3, but it is not as variable in X-rays) or possibly a millisecond pulsar (e.g. PSR $1023+0038)$, but no spin period has been detected to date.

The SALT spectra were obtained during $\sim 1 \mathrm{~h}$ tracks, with $300 \mathrm{~s}$ repeat exposures. They show significant changes, often over the timescale of an exposure, sometimes exhibiting weak emission lines of the Balmer series, HeI and HeII, and other time showing Balmer and other absorption features, as can be seen in Fig. 8 . Radial velocity variations of the combined spectra indicate a possible $\sim 7 \mathrm{~h}$ orbital period, which is under investigation. The high speed photometry also shows variations on a similar timescale, but with periods of enhanced flickering possibly occurring at the same orbital phase.

\section{Future Plans}

In 2014 it is planned to extend the SALT observational work on CVs and related objects to include time resolved polarimetric imaging and spectropolarimetry. Polarized continuum flux variations will be used to probe the cyclotron emission, for example using the Stokes imaging technique (see Potter et al. 2004). This will be combined with Doppler and Roche tomography and eclipse mapping to assist in building detailed models of $\mathrm{mCVs}$, and may include using relevant particle or MHD codes.

Following the launch of the Indian X-ray satellite, ASTROSAT (in 2014), it is planned to begin coordinated observations using SALT to undertake contemporaneous optical observations of mCVs, LMXBs and related objects. ASTROSAT will have both, hard and soft X-ray instruments, the former with excellent sensitivity and energy range, plus a UV imaging telescope. Adding such data will allow for a comprehensive multiwavelength analysis of these systems. 


\section{Acknowledgement}

The work summarized in this paper represents a number of collaborative SALT projects with various colleagues, including Jean Marc Bonnet-Bidaud, Hannes Breytenbach, Domitilla de Martino, Marissa Kotze, Darragh O'Donoghue and Stephen Potter, to name a few. The research of the author is supported by NRF rated researcher and incentive funding awards. The observations presented were carried out using the Southern African Large Telescope.

\section{References}

[1] Bonnet-Bidaud, J.M., et al.: 2012, Mem. S.A. It., 83,742 .

[2] Bridge, C.M., et al., 2004, MNRAS, 351, 1423. doi:10.1111/j.1365-2966.2004.07884.x

[3] Buckley, D.A.H., Swart, G.P \& Meiring, J.G., 2006, Proc. SPIE, 6267, $62670 Z$. doi:10.1117/12.673750

[4] Buckley, D.A.H., et al., 2010, Poprc. SPIE, 7735, 77359-1.
[5] Butters, O.W., et al., 2008, A\&A, 487, 271.

[6] Kotze, E. \& Potter, S.B., 2013, these proceedings.

[7] Larsson, S., 1989, A\&A, 217, 146.

[8] Michaud, C., et al., 2012, Mem. S.A. It., Vol. 83, 665.

[9] O'Donoghue, D., et al., 2006, MNRAS, 372, 151. doi:10.1111/j.1365-2966.2006.10834.x

[10] Potter, S.B., et al., 2004, MNRAS, 348, 316. doi:10.1111/j.1365-2966.2004.07379.x

[11] Welsh, B., et al., 2011, IAU Symp. 285, 99.

\section{DISCUSSION}

PAULA SZKODY: Can you give more information on the QPO in EX Hya? Is it seen in the lines or continuum?

DAVID BUCKLEY: The QPO was detected using SALT and BVIT with a broad-band $R$-filter, so includes both continuum and $\mathrm{H} \alpha$. The characteristic period was $\sim 100 \mathrm{~s}$, or more likely, its harmonic. 